\section{Adjudicación de Derechos Sociales en la Constitución de Transformación Social de Sudáfrica ${ }^{1}$}

Sandra Liebenberg*

\section{Introducción}

Uno de los temas más controvertidos de la creciente jurisprudencia sobre derechos sociales de Sudáfrica se refiere a la forma en que los tribunales debieran hacer cumplir las obligaciones que imponen esos derechos. El debate se ha centrado particularmente en determinar hasta qué punto los tribunales deben afirmar que existe un derecho ejecutable (o justiciable) a la satisfacción de necesidades básicas por parte de quienes carecen de los medios necesarios para procurárselas. En el contexto sudafricano, este problema afecta a una parte importante de la población y debe, además, ser contextualizado dentro del alto grado de desigualdad que marca a esta sociedad ${ }^{2}$.

La Constitución de Sudáfrica se describe, en términos generales, como una Constitución de transformación social ${ }^{3}$. A diferencia de muchas constituciones clásicas de corte liberal, su principal objetivo no radica en limitar el poder estatal, sino en facilitar un cambio fundamental en las injustas relaciones políticas, económicas y sociales existentes en Sudáfrica ${ }^{4}$. De ahí que el Preámbulo de la Constitución proclame que fue aprobada "con el fin de sanar las divisiones del pasado y establecer una sociedad basada en valores democráticos, justicia social y derechos humanos fundamentales". Los principios básicos de la Constitución se refieren al "logro de la igualdad", a la "eliminación del racismo y el sexismo", y a un sistema de gobierno democrático con rendición de cuentas, que sea receptivo y abierto $^{5}$.

El compromiso con la justicia social es un aspecto esencial de los objetivos de transformación y los procesos de nuestra Constitución y debe inspirar la interpretación de la Declaración de Derechos. En su Cuarta Conferencia Bram Fischer Memorial, Dikgang Moseneke, Presidente de la Corte Suprema, describió el importante papel de la justicia social en la adjudicación constitucional:

“...[S]e sostiene aquí que una jurisprudencia creativa sobre la igualdad, combinada con una interpretación sustantiva del contenido de los derechos "socioeconómicos", debiera restablecer a la justicia social como el principal valor fundacional de nuestra democracia constitucional a la par, si no interactivamente, con la dignidad humana, la igualdad, la libertad, la rendición de cuentas, la receptividad y la apertura" 6 .
* Cátedra H. F. Oppenheimer de Derechos Humanos, Departamento de Derecho Público, Universidad Stellenbosch.

** Texto traducido al castellano por Jennifer Metcalfe y revisado por el Centro de Derechos Humanos.
Ver notas del texto a partir de la página 67. Este artículo se encuetra disponible en www.anuariocdh.uchile.cl 


\section{Justicia Social, Democracia y Adjudicación}

La inclusión de una serie de derechos socioeconómicos como derechos justiciables en la Constitución de 1996 puede ser vista como una lucha exitosa de varios actores políticos y organizaciones de la sociedad civil en cuanto consagra constitucionalmente la obligación del Estado de satisfacer estas necesidades ${ }^{7}$. Al obligar constitucionalmente al Estado a que garantice que todos "tengan acceso" a una serie de derechos socioeconómicos, la satisfacción de los derechos en cuestión queda claramente reconocida como un hecho público y no se la relega a la esfera meramente "privada" o del mercado ${ }^{8}$.

La distinción entre derechos civiles y políticos "justiciables" versus derechos socioeconómicos no justiciables es, en sí, una cuestión profundamente política. Esto privilegia la libertad negativa y el statu quo económico existente, además de ocultar el costo y las dimensiones de políticas públicas aplicadas en los derechos civiles y políticos ${ }^{9}$. En las democracias constitucionales, donde la adjudicación es un elemento importante de las estructuras fundamentales de gobierno de un país, la exclusión o debilidad en el cumplimiento de los derechos socioeconómicos puede marginar los intereses de los desposeídos, además de ocultar las barreras socioeconómicas que impiden lograr relaciones sociales más igualitarias ${ }^{10}$. Por el contrario, la inclusión de derechos sociales transforma el tema de las necesidades no cubiertas en una cuestión de derechos ${ }^{11}$.

En los primeros debates sobre derechos sociales, Nicolas Haysom aportó una fundamentación desde la perspectiva de la democracia participativa para la inclusión de una base de derechos sociales justiciables en la Constitución:

"Al plasmar en la Constitución ciertos derechos socioeconómicos, la sociedad los está elevando a la categoría de condición necesaria para el ejercicio de un mínimo de igualdad cívica. En consecuencia, se establecen las condiciones para la democracia, mediante el uso eficaz de los derechos civiles y políticos... Este documento no va más allá que plantear que la Constitución debiera garantizar un mínimo de derechos para enriquecer la contienda política y la participación democrática, sin restringir las opciones políticas, sino facilitando la participación genuina en los derechos sociales y políticos ${ }^{\prime 12}$.

\section{III. ¿Hacia una Jurisprudencia de Transformación Social en el ámbito de los Derechos Sociales?}

\section{La jurisprudencia constitucional de Sudáfrica en el ámbito de los dere- chos sociales.}

a) Revisión de la razonabilidad de los derechos positivos

En los tres primeros casos de derechos socioeconómicos sometidos a su consideración (Soobramoney, Grootboom y TAC), el Tribunal Constitucional se vio directamente enfrentado al desafío de desarrollar 
un modelo para hacer cumplir las obligaciones positivas que imponen las Secciones 26 y 27. El Tribunal Constitucional rechazó la idea de que estas disposiciones asignan al Estado una obligación directa e ilimitada de proporcionar recursos sociales y servicios a la gente mediante la sola demanda, en el contexto de los argumentos planteados por las intervenciones de los amici curiae en los casos Grootboom y TAC. Los amici curiae intentaron persuadir al Tribunal Constitucional de que adoptara la idea de obligaciones fundamentales mínimas como la desarrollada por el Comité de Naciones Unidas sobre Derechos Económicos, Sociales y Culturales ${ }^{13}$. El Tribunal rechazó una interpretación de los derechos socioeconómicos que "pudiese dar origen a un derecho positivo autónomo e independiente, que pueda hacerse efectivo por la justicia sin tomar en cuenta las consideraciones de la segunda subsección de las secciones 26 y $27^{\prime \prime 14}$. De ahí que el alcance de los deberes positivos del Estado estén limitados por los siguientes tres elementos clave: (a) la obligación de "adoptar medidas razonables de índole legislativa y de otra naturaleza"; (b) "lograr la realización progresiva" del derecho, y (c) "dentro de los recursos disponibles"15.

El Tribunal señaló una serie de inquietudes relacionadas con el concepto de obligaciones básicas mínimas. En primer lugar, identificó el problema de definir el "mínimo básico", dado que la situación de cada grupo es diferente como también lo son sus necesidades sociales ${ }^{16}$. En segundo lugar, señaló que la imposición de un mínimo básico impone obligaciones poco realistas al Estado, ya que "es imposible dar acceso a todos, ni siquiera a un servicio básico, de manera inmediata"17. Por último, el Tribunal Constitucional sostuvo que el mínimo básico era incompatible con las competencias institucionales y el papel de los tribunales ${ }^{18}$. Sin embargo, manifestó que, en un caso específico, la evidencia puede demostrar que existe un mínimo básico de un servicio determinado que debiera ser tomado en cuenta a la hora de determinar si las medidas adoptadas por el Estado son razonables ${ }^{19}$.

El Tribunal procedió a desarrollar un modelo de "revisión de razonabilidad" para la adjudicación de prestación de servicios sociales y de recursos. Al revisar las obligaciones positivas que las disposiciones sobre derechos sociales imponen al Estado, la pregunta principal del Tribunal Constitucional es si es razonable suponer que las medidas elegidas son capaces de permitir la satisfacción de los derechos en cuestión ${ }^{20}$. El Tribunal ha tomado la precaución de recalcar que se otorga un amplio margen de acción a los organismos políticos del gobierno para que tomen las decisiones adecuadas en materia de políticas y que el papel del Tribunal consiste en determinar si dichas políticas caen dentro de los límites de lo "razonable" 21 . La razonabilidad de las políticas sociales estatales se evalúa principalmente en términos de su capacidad de inclusión (ya que ellas deben atender a las necesidades de los grandes grupos sociales) y también se evalúa en cuanto al corto, mediano y largo plazo $^{22}$. Aquí, una revisión de la razonabilidad parece una cuestión formalista y abstracta que equipara las necesidades de los ricos con los pobres y exige que el gobierno atienda a ambos grupos por igual. Sin 
embargo, el Tribunal reconoce que "los pobres son especialmente vulnerables y sus necesidades requieren de atención especial"23. La razonabilidad no se evalúa simplemente por el avance estadístico en facilitar el acceso a los diferentes derechos socioeconómicos, sino también por intereses relativos a la dignidad del grupo afectado, especialmente por el impacto de la denegación de derechos específicos a los demandantes. En el caso Grootboom el Tribunal Constitucional sostuvo que:

"La razonabilidad debe ser entendida también en el contexto global de la Declaración de Derechos. El derecho a tener acceso a una vivienda adecuada está firmemente arraigado, porque valoramos a los seres humanos y deseamos garantizarles sus necesidades humanas básicas. Una sociedad debe tratar de garantizar que las necesidades vitales básicas de todos sus integrantes estén satisfechas para poder ser una sociedad basada en la dignidad humana, la libertad y la igualdad. Para ser razonables, las medidas no pueden dejar de lado el grado y alcance de la denegación del derecho que pretenden hacer realidad. Aquellos cuyas necesidades son más urgentes y que, en consecuencia, corren mayor peligro de no llegar a gozar de todos los derechos, no deben ser ignorados por las medidas propuestas para alcanzar la realización del derecho... Si las medidas, aunque sean exitosas en términos estadísticos, no logran responder a las necesidades de quienes están más desesperados, no podrán pasar la prueba" ${ }^{24}$.

El estándar de revisión de la razonabilidad requiere que los programas de gobierno cuenten con disposiciones razonables de corto plazo para aquellos cuyas circunstancias socioeconómicas sean apremiantes o intolerables. En el caso Grootboom la falta de un programa que atendiera las necesidades inmediatas de vivienda se consideró injusta $y$, en consecuencia, no coherente con la Sección $26^{25}$. En el caso TAC, la rigidez de las políticas del gobierno, al negarse a distribuir la droga antirretroviral Nevirapina para reducir la transmisión del VIH de madre a hijo más allá de los 18 sitios piloto, se consideró no razonable. Sencillamente, no había una justificación razonable para negar un "tratamiento médico simple, económico y que posiblemente podía salvar la vida" ${ }^{26}$ de mujeres pobres y sus bebés recién nacidos en el sector de salud pública ${ }^{27}$. El Tribunal recalcó que su fallo no implicaba que "todos pueden reclamar acceso inmediato a dicho tratamiento", si bien "el ideal... es alcanzar esa meta" ${ }^{28}$.

En ambos casos, el Tribunal sometió a un estricto escrutinio las justificaciones que el gobierno entregó por no haber tomado las medidas requeridas para satisfacer necesidades básicas. Las justificaciones, fundadas en cuestiones de recursos y políticas públicas, fueron consideradas no convincentes. En el caso Grootboom, el Tribunal se limitó a emitir una orden ${ }^{29}$, mientras que en el caso TAC, el Tribunal dispuso medidas de asistencia de vasto alcance, imponiendo al gobierno que distribuyera la Nevirapina y que ampliara la disponibilidad de servicios de exámenes de laboratorio y de orientación en hospitales y clínicas del sector de salud pública, en general, para reducir la transmisión del VIH 
de madres a hijos ${ }^{30}$. Sin embargo, el Tribunal tomó la precaución de aclarar que las disposiciones pertinentes en materia de derechos socioeconómicos no dan origen a un derecho personal ejecutable individualmente respecto de estos recursos y servicios socioeconómicos ${ }^{31}$.

\section{b) Intersección de los derechos sociales con los derechos de igualdad}

El segundo tipo de situación en que el Tribunal ha debido resolver sobre las obligaciones positivas impuestas por los derechos sociales se refiere a la promulgación de legislación excluyente sobre beneficios sociales. Esto queda claramente ejemplificado en la decisión del Tribunal Constitucional en los casos "Khosa con Ministerio de Desarrollo Social" y "Mahalaule con Ministerio de Desarrollo Social" (en adelante "Khosa") ${ }^{32}$. El caso trata sobre la impugnación de la validez de la asistencia social que limita la elegibilidad de los subsidios sociales a ciudadanos sudafricanos $^{33}$. El Tribunal sostuvo que la exclusión de los residentes permanentes de la legislación sobre ayuda asistencial constituía tanto una limitación del derecho de acceso a la asistencia social (Sección 27) como al derecho contra la discriminación injusta (Sección 9). Afirmó, además, que el requisito que exige que tanto el adulto principal encargado del cuidado del niño como el niño mismo, sean ciudadanos sudafricanos para ser acreedores de la asignación a favor de la infancia, "socava" los derechos socioeconómicos de los niños en virtud de la Sección 28(1)(c) de la Constitución ${ }^{34}$.

Luego de analizar detalladamente la razonabilidad del esquema legislativo en los términos de la Sección 27(2), el Tribunal consideró que las consideraciones financieras presentadas por el Estado para limitar el acceso a las subvenciones sociales de los ciudadanos no eran convincentes ${ }^{35}$. También rechazó el razonamiento del Estado en sentido de que la exclusión de los residentes permanentes de los beneficios sociales era legítima, puesto que impedía que se convirtieran en una "carga" para el Estado y, en consecuencia, estimulaba a los extranjeros a volverse autosuficientes. Según el Tribunal, la inmigración debía controlarse "por otros medios que no fueran permitir que los inmigrantes se radicaran aquí en forma permanente, para luego abandonarlos a la indigencia cuando se encontraran frente a tiempos difíciles" ${ }^{\prime 36}$. Hay otros métodos menos severos para reducir el riesgo de que los residentes permanentes se transformen en "una carga" para el Estado y que no implican su exclusión del acceso a la asistencia social ${ }^{37}$. En esta evaluación de la razonabilidad de las dos justificaciones presentadas por el Estado, es bien evidente la aplicación de un test de proporcionalidad. En última instancia, el impacto que tiene la exclusión de la asistencia social en la vida y la dignidad de los residentes permanentes superó las consideraciones financieras y de inmigración en que se apoyaba el Estado ${ }^{38}$.

El riguroso estándar de análisis aplicado en este caso debe entenderse en el contexto de la denegación de un beneficio social básico a un grupo vulnerable, y de las intersecciones entre violaciones a un derecho 
socioeconómico y al derecho contra la discriminación injusta ${ }^{39}$. Al haber encontrado violaciones de las Secciones 9 y 27, el Tribunal reconoció la dificultad de aplicar un análisis de cláusula limitativa a los derechos socioeconómicos en las Secciones 26 y $27^{40}$. Sostuvo que no era necesario decidir si se requería un grado diferente de razonabilidad en relación con la Sección 36. Si bien se supuso que este era el caso, el Tribunal concluyó que la exclusión de los residentes permanentes de la asistencia social "no es razonable ni justificable conforme al significado de la Sección 36"41. El Tribunal dispuso de un categórico remedio legal al interpretar que los residentes permanentes estaban incluidos entre los requisitos de elegibilidad de la legislación ${ }^{42}$.

\section{C) Revisión de la privación del acceso ya existente}

La tercera situación considerada por los Tribunales se refiere a grupos privados del acceso a derechos sociales del que ya gozaban ${ }^{43}$.

El Tribunal Constitucional ha caracterizado estas situaciones como violaciones a las obligaciones "negativas" impuestas por los derechos sociales $^{44}$. Se han presentado principalmente en el contexto del desalojo de personas de sus viviendas, reforzadas por la garantía explícita que otorga la Sección $26(3)^{45}$. Gran parte de la jurisprudencia se ha referido a la interpretación de piezas clave de la legislación promulgada para poner en vigencia la Sección 26(3), especialmente la Ley sobre Prevención de Desalojo Ilegal y de Ocupación Ilegal de Tierras (Prevention of Ilegal Eviction and Unlawful Occupation of Land Act 19 of 1998 -"PIE") $)^{46}$.

La decisión más importante sobre la interpretación de PIE es la del Tribunal Constitucional en el caso Municipalidad de Port Elizabeth con Varios Ocupantes ("PE Municipality") $)^{47}$. La sentencia ilustra cómo se debe interpretar PIE para promover los objetivos y valores contenidos en la Sección 26(3). El caso se refiere al desalojo de unas 68 personas, efectuado por la Municipalidad de Port Elizabeth, que habitaban chozas construidas en tierras de propiedad privada dentro de la jurisdicción de la Municipalidad. La mayoría había llegado a estos sitios eriazos luego de ser desalojados de otros terrenos. Al emitir el requerimiento, la Municipalidad actuaba en respuesta a una petición de la comunidad. El Tribunal señaló que la Sección 26(3) reconoce que "se puede realizar el desalojo de gente que vive en asentamientos informales aun cuando ocasione la pérdida del hogar" ${ }^{48}$. Sin embargo, luego señala que, en general, "un tribunal debe ser reacio a conceder el desalojo de ocupantes medianamente asentados, a menos que se disponga de una alternativa razonable, aunque sea como medida provisional, en tanto se accede a una vivienda del programa habitacional formal"49. Por lo tanto, para demostrar al Tribunal que un desalojo es "justo y equitativo" los organismos estatales tendrán que demostrar que se consideró muy seriamente la posibilidad de proporcionar una vivienda alternativa a los ocupantes ${ }^{50}$. El Tribunal indicó también que, salvo por circunstancias especiales, "normalmente, no sería justo ni equitativo ordenar el desalojo sin realizar una discusión adecuada y, cuando proceda, una 
mediación" ${ }^{\prime 51}$. El punto crítico que indicó la jueza Sachs en su fallo es que en el choque entre los derechos de propiedad y "la verdadera desesperación de gente que tiene una necesidad extrema de contar con un lugar para vivir", los tribunales no debieran privilegiar automáticamente los derechos de propiedad ${ }^{52}$. Su papel, más bien, es encontrar una solución justa y equitativa en el contexto de los factores específicos que son pertinentes en cada caso en particular. El fallo en el caso de la Municipalidad de Port Elizabeth ciertamente contempla circunstancias en las cuales se puede desalojar a las personas de sus hogares. Estas podrían incluir, por ejemplo, situaciones en las que la gente se toma terrenos intencionalmente con el fin de alterar los programas organizados de vivienda y colocarse en primer lugar para recibir el beneficio habitacional ${ }^{53}$. Si bien la entrega de una vivienda alternativa adecuada no es un requisito absoluto, no deja de ser una consideración de peso en la evaluación de si una orden de desalojo es "justa y equitativa" en determinadas circunstancias ${ }^{54}$.

El fallo reciente en el caso Presidente de la República de Sudáfrica y Otro con Modderklip Boerdery (Pty) Ltd ("Modderklip") $)^{55}$ se refiere a la interpretación de las obligaciones del Estado en el contexto de los esfuerzos infructuosos de un terrateniente por hacer cumplir una orden de desalojo otorgada en virtud de PIE contra una comunidad que ocupaba sus tierras. Se estima que, cuando el terrateniente intentó hacer cumplir el fallo, la comunidad constaba de aproximadamente 40.000 personas, un tercio de las cuales supuestamente eran inmigrantes ilegales ${ }^{56}$. El receptor del Tribunal Supremo exigió al propietario un depósito de 1,8 millones de rands para garantizar el costo del desalojo, monto que excedía el valor de las tierras. Luego del fracaso de sus esfuerzos por lograr el apoyo de diversos organismos estatales para ejecutar el fallo, solicitó al Tribunal que ordenara al Estado que lo ayudara en la reivindicación de sus derechos de propiedad, en el marco de la Constitución. El Tribunal Constitucional no estimó necesario resolver el caso sobre la base de los derechos de propiedad del terrateniente (conforme a la Sección 25) ni de los derechos a vivienda de los ocupantes (Sección 26) ${ }^{57}$. En cambio, sostuvo que el hecho de que el Estado no hubiera tomado medidas razonables para ayudar al terrateniente a revindicar su propiedad, junto con evitar el desorden social en gran escala que ocasionaría el desalojo de una numerosa comunidad que no tenía dónde ir, constituía una violación del principio del estado de derecho consagrado en la Sección 1 (c) como también del derecho de acceso a los tribunales y otros foros independientes conforme a lo establecido en la Sección 34 de la Constitución ${ }^{58}$. El Tribunal aseveró que la realización progresiva del derecho de acceso a la vivienda o a tierras para quienes carecen de hogar requiere de una "cuidadosa planificación", "procedimientos justos" y "procesos ordenados y previsibles" 59 . La ocupación de tierras debe ser desalentada en toda circunstancia ${ }^{60}$. Al mismo tiempo, estas medidas no se considerarán razonables si no dejan "lugar alguno para realizar ajustes relativamente menores a la luz de la realidad cambiante" ${ }^{\prime 61}$. La reparación novedosa otorgada por el Tribunal fue exigir que el Estado indemnizara 
al terrateniente por la ocupación ilegal de su propiedad. Es importante mencionar que el fallo señala expresamente que "los residentes están autorizados para ocupar las tierras hasta que se les hayan proporcionado terrenos alternativos, ya sea por el Estado, las autoridades provinciales o las autoridades locales" ${ }^{\prime 62}$.

La resolución del Tribunal en "Jaftha con Schoeman" y "Van Rooyen con Stolz y Otros" ("Jaftha") ${ }^{63}$ representa un avance significativo en el enfoque del Tribunal en el examen de las obligaciones que imponen los derechos sociales. El caso cuestionaba la constitucionalidad de las resoluciones de la Ley del Tribunal de Primera Instancia, que permitió la venta de las casas de unas mujeres para cubrir unas deudas insignificantes. Las demandantes, dos mujeres de escasos recursos, eran propietarias de viviendas adquiridas mediante subsidios estatales. Al caer en mora por deudas mínimas (p. ej. la compra de vegetales) se falló en su contra en un juicio y sus viviendas salieron a remate. Como resultado de esta venta forzosa, los habitantes fueron desalojados. También era común que, una vez desalojados, los habitantes no contaran con una vivienda alternativa ${ }^{64}$. El Tribunal Supremo había manifestado que la pérdida del derecho de las demandantes de ocupar sus casas no fue causada por la venta forzosa autorizada por el fallo del juicio ejecutivo porque, si el deudor hubiera optado por mantener la ocupación de la vivienda después de la venta forzada, la Prevención del Desalojo Ilegal se aplicaría a la demanda presentada por el comprador de la propiedad. El juicio ejecutivo, si bien pone fin a la propiedad del deudor, no constituye una violación de la Sección 26 en cuanto esta disposición no autoriza a nadie a apropiarse de una vivienda o a ocupar una unidad habitacional específica ${ }^{65}$.

Por otra parte, el Tribunal Constitucional caracterizó las disposiciones del fallo como una autorización de una violación negativa de la Sección 26(1) al permitir que "una persona sea privada del derecho de acceso existente a una vivienda adecuada"66. Esta obligación negativa no está sujeta a las restricciones establecidas en la subsección (2) relativas a la limitación de recursos y la realización progresiva. Al privar a las personas del acceso existente a la vivienda ( $y$, en consecuencia, a otros derechos socioeconómicos), se limitan sus derechos, hecho que no se justifica en términos de los exigentes requisitos de la cláusula limitativa general (Sección 36), incluido el requisito de la ley de aplicación general ${ }^{67}$. El Tribunal expresamente no entró en detalles acerca de las circunstancias que implicarían una violación de las obligaciones negativas que impone la Constitución ${ }^{68}$.

El Tribunal no encontró justificación alguna para las desmesuradas disposiciones de la Ley del Tribunales de Primera Instancia en cuanto a la cláusula limitativa general ${ }^{69}$. Como forma de reparación, hizo aplicables al caso las disposiciones legales que exigen la supervisión judicial de la ejecución contra los bienes muebles de los deudores, tomando en consideración todas las "circunstancias pertinentes"70. Entre los principales factores pertinentes al ejercicio de esta supervisión judicial 
debe considerarse si una orden que autoriza la venta forzosa es "extremadamente desproporcionada".

“Este sería el caso si los intereses del acreedor en el juicio por obtener el pago fueran considerablemente inferiores a los intereses del deudor respecto de la seguridad de la tenencia de su vivienda, particularmente si la venta de la vivienda dejará al deudor y a su familia carentes de ella"71.

Otra consideración consiste en encontrar "alternativas creativas" que permitan recuperar lo adeudado, pero que recurren a la venta forzada de la vivienda del deudor "solamente como último recurso"72. En consecuencia, el hecho de que una persona pueda verse privada de su vivienda es una consideración de peso en la supervisión judicial de ventas forzosas.

Si bien el Tribunal no ha llegado al extremo de reconocer el derecho irrestricto a la vivienda alternativa en los casos de desalojo, ha requerido una seria consideración del impacto del desalojo y la disponibilidad de alternativas viables con el fin de evitar la falta de vivienda. Asimismo, lo anterior ilustra que, tratándose de la vivienda, ya no se privilegian automáticamente los derechos de propiedad. La necesidad de vivienda de los sectores pobres y vulnerables a la falta de hogar constituye ahora una consideración altamente pertinentes en casos en los cuales la gente está expuesta al desalojo.

Evaluaremos a continuación el potencial transformador de esta jurisprudencia dentro de las perspectivas teóricas desarrolladas anteriormente.

\section{Potencial transformador de la jurisprudencia}

Es importante reconocer que el examen de la razonabilidad puede llegar a representar un estándar de examen muy deferente. Dennis Davis sostiene que los Tribunales pueden modelar el concepto de grado de razonabilidad "de modo que, a veces, puede parecer una prueba de racionalidad y garantiza que el Tribunal pueda evitar todo enfrentamiento potencial con temas de política socioeconómica"73. El peligro radica en que la revisión de la razonabilidad se convierte en un sustituto para que los Tribunales apoyen el punto de vista del Estado en cuanto a justificar sus propias políticas ${ }^{74}$.

Sin embargo, también se ha sostenido que la revisión de la razonabilidad tiene la ventaja de ser un modelo flexible y sensible al contexto para el examen de las demandas de derechos socioeconómicos ${ }^{75}$. Por lo tanto, la posición del Tribunal en el caso Grootboom fue que la razonabilidad de un conjunto de medidas destinadas a hacer efectivos los derechos socioeconómicos específicos debe evaluarse a la luz de su contexto social, económico e histórico como también en el marco global de la Declaración de Derechos ${ }^{76}$. En este sentido, el "examen de razonabilidad" evita cerrar el análisis y genera la posibilidad permanente de cuestionar las privaciones socioeconómicas en el marco de contextos cambiantes. En consecuencia el "examen de razonabilidad" puede facilitar la creación 
de un espacio de participación y diálogo en torno a las demandas sociales. Esto queda ejemplificado por la manera en que la Campaña de Acción para el Tratamiento logró recurrir al examen de razonabilidad para obtener un gran triunfo en la entrega de tratamiento médico adecuado para reducir el riesgo de transmisión del VIH de madre a hijo ${ }^{77}$. Este triunfo constituyó un avance significativo en la estrategia más amplia de transformación de TAC destinada a alcanzar un programa general antirretroviral anunciado por el gobierno en agosto de 2003 para personas portadoras de VIH/ SIDA. TAC y otras organizaciones de la sociedad civil pudieron utilizar los criterios para un programa razonable que fueron establecidos en el caso Grootboom y en el caso de transmisión de madre a hijo en acciones generalizadas de promoción, defensa y apoyo para un programa antirretroviral general ${ }^{78}$.

\section{Realización del potencial transformador de los derechos sociales}

En vista de la acumulación de injusticias históricas, la plena realización de los derechos sociales requerirá de profundos cambios estructurales con el tiempo. ¿Podrán los Tribunales desempeñar un papel importante para facilitar estos cambios fundamentales? Mi visión personal es que, en la actual jurisprudencia sobre derechos sociales de los Tribunales, hay un potencial considerable para la transformación. Sin embargo, la realización de este potencial depende de que los Tribunales interpreten los derechos sociales en forma sustantiva. Puedo señalar cuatro áreas en las cuales la interpretación que hagan los Tribunales de los derechos sociales puede facilitar la transformación.

\section{a) Revisión substantiva de la razonabilidad}

En primer lugar, las demandas por privación de necesidades básicas debieran provocar un alto grado de análisis judicial. Me he referido a la tensión existente en la interpretación judicial de las necesidades básicas entre, por una parte, apoyar un espacio más participativo y que fomente el diálogo, evitando interpretaciones excesivamente rígidas y limitadas $y$, por otra, la importancia de articular obligaciones claras con el fin de asegurar la satisfacción de las necesidades urgentes de la gente. Esto se puede lograr dentro de una estructura de revisión de la razonabilidad desarrollada por el Tribunal, que tome en cuenta las condiciones del contexto.

Al evaluar la razonabilidad de las acciones u omisiones del Estado, la principal consideración debiera ser la posición del demandante en la sociedad, la historia y la naturaleza de la privación sufrida, así como su impacto sobre el sujeto y sobre otros que se encuentran en una situación similar $^{79}$. Un enfoque específico de tal investigación debiera ser el impacto que tales privaciones han tenido en la capacidad de los grupos afectados para participar como pares en la sociedad. Debe prestarse especial atención a la interacción entre los obstáculos a la paridad participativa identificados por Fraser $^{80}$, a saber, la falta de acceso a recursos económicos y sociales, el estigma social y los estereotipos que van asociados con la 
pobreza y su interacción con otras formas de injusticia en reconocer derechos e identidades, tales como la raza, el género y la orientación sexual. Al considerar las justificaciones del Estado por no lograr satisfacer las necesidades de subsistencia, los Tribunales se verían en la necesidad de realizar un riguroso análisis de proporcionalidad (bastante similar al enfoque utilizado por el Tribunal en el caso Khosa). En este caso, los Tribunales están en una buena posición para destacar el impacto de las macro injusticias que afectan a demandantes específicos en situaciones concretas. La jueza Sachs describe el deber de los Tribunales en cuanto a tratar de lograr justicia para los litigantes en una realidad sistémica de desigualdad social:

"Las injusticias heredadas a nivel macro inevitablemente dificultarán la labor de los tribunales para garantizar la equidad a nivel micro de modo inmediato. El Poder Judicial no puede, por sí solo, corregir todas las injusticias sistemáticas existentes en nuestra sociedad. Pero al menos puede efectivamente suavizar y minimizar el grado de injusticia e inequidad que acarrea el desalojo de los más débiles que viven en condiciones de desigualdad" ${ }^{81}$.

Es importante, además, que la evaluación de la "razonabilidad" en los casos de derechos socioeconómicos se base en el requisito de "realización progresiva" señalado en las secciones 26(2) y 27(2). Este concepto, tomado del Artículo 2 del Pacto Internacional de Derechos Económicos, Sociales y Culturales, implica un proceso dinámico en el cual los niveles de prestaciones sociales mejoran con el tiempo ${ }^{82}$. En Grootboom, el Tribunal apoyó la opinión del Comité de Derechos Económicos, Sociales y Culturales, en el sentido de que la "realización progresiva impone la obligación de encaminarse con la mayor rapidez y eficacia posible" hacia el objetivo final de la plena realización. También respaldó la opinión de este Comité en cuanto a que "las medidas deliberadamente retrógradas" requieren de justificación y un examen minucioso ${ }^{83}$.

El verdadero desafío de los litigios por derechos sociales estriba en situaciones de grandes grupos que actualmente están excluidos de las prestaciones sociales, lo cual está ejemplificado por el actual sistema de seguridad social de Sudáfrica. La cobertura abarca a quienes cuentan con empleo formal, a través del sistema de seguro social ${ }^{84}$, que incluye el pago de subvenciones sociales con fondos públicos a ciertos grupos focalizados (niños, ancianos y discapacitados) ${ }^{85}$. Sin embargo, no se proporciona asistencia social alguna a niños mayores de 14 años ${ }^{86}$ ni a mujeres menores de 60 y hombres menores de 65 años que viven en situación de pobreza que estén afectados por una cesantía estructural de largo plazo ${ }^{87}$. Para este grupo (que alcanza un total de aproximadamente 8,4 millones de personas) ${ }^{88}$, el derecho consagrado en la Sección 27(1)(c) de la Constitución ${ }^{89}$ es casi inexistente ${ }^{90}$. Queda por verse si los tribunales, frente a un caso pertinente, exigirán que el Estado adopte medidas positivas para salvar este vacío en la seguridad social. En este contexto cobra importancia la obligación del Estado, reconocida por el Comité de Derechos Económicos, Sociales y Culturales de Naciones Unidas, de 
formular e implementar una estrategia nacional y un plan de acción para abordar el tema del acceso a los derechos socioeconómicos a toda la población. Dicha estrategia y plan de acción deben ser formulados y revisados periódicamente mediante un proceso participativo y transparente, que incluya indicadores y puntos de referencia que permitan seguir de cerca el avance del mismo ${ }^{91}$. Aun cuando los tribunales no pueden emitir órdenes para un cierre inmediato de la brecha existente en la atención de seguridad social, al menos pueden exigir que la planificación sea participativa y que se adopten medidas concretas para la plena realización de este importante derecho social.

Se necesitará una fiscalización constante para garantizar que la revisión de la razonabilidad no degenere y se transforme en una norma excesivamente deferente. El colocar a los demandantes junto con la naturaleza y la historia de la privación que han sufrido al centro de la investigación sobre razonabilidad ayudará a concentrarse en las barreras sociales y económicas para lograr una sociedad más igualitaria.

\section{b) Medidas enérgicas}

En segundo lugar, los tribunales tienen a su alcance facultades suficientes para otorgar soluciones más eficaces en los casos de derechos sociales. Así, por ejemplo, los Tribunales pueden exigir que el Estado lleve a cabo un plan o programa que permita lograr los cambios necesarios y que adopte medidas concretas y focalizadas conforme a dicho plan ${ }^{92}$. En este contexto, el Tribunal debe ser capaz de superar su reticencia a ordenar medidas de supervisión* con el fin de facilitar las reformas estructurales a largo plazo necesarias para la satisfacción de los derechos socioeconómicos ${ }^{93}$. Las medidas de supervisión tienen un amplio potencial no sólo para que los Tribunales fiscalicen la implementación de tales órdenes, sino también para realzar la participación de la sociedad civil y de las instituciones estatales que respaldan a la democracia constitucional en los litigios sobre derechos socioeconómicos. Los Tribunales también pueden aliviar concretamente a quienes estén experimentando privaciones, evitando de ese modo daños irreparables a la vida, la salud y el desarrollo futuro. La naturaleza y alcance de esta ayuda dependerán de las circunstancias, pero deberán reflejar la convicción, manifestada en Grootboom, de que una sociedad "debe intentar garantizar que las necesidades vitales básicas de todos sus integrantes estén cubiertas para lograr ser una sociedad basada en la dignidad humana, la libertad y la igualdad"94.

Mediante la aplicación de un grado superior de revisión y medidas enérgicas en casos en que el problema es la insatisfacción de necesidades básicas, los Tribunales pueden crear conciencia pública acerca del impacto y las consecuencias sociales de la pobreza. La transformación se promueve cuestionando la distribución desigual de la riqueza y reafirmando el derecho a beneficios sociales y económicos cuando previamente no existía tal reconocimiento ${ }^{95}$. 


\section{c) Un discurso de transformación social}

En tercer lugar, los tribunales pueden contribuir a la transformación a través de la naturaleza de su discurso en los fallos sobre derechos socioeconómicos. Este papel retórico es importante incluso cuando los tribunales se sienten limitados por las políticas institucionales para emitir una orden judicial que pueda tener un amplio impacto en las asignaciones presupuestarias. En consecuencia, los tribunales pueden resistir la tentación de limitarse a las necesidades menores y esforzarse por exponer las situaciones subyacentes de injusticia social que dan origen a las carencias en cuestión. La resolución de la jueza Sachs, en el caso de la Municipalidad de Port Elizabeth, constituye un ejemplo excelente de la forma en que los tribunales de justicia pueden involucrase con los factores históricos, socioeconómicos, políticos y legales que subyacen a un caso de desalojo de gente pobre en Sudáfrica ${ }^{96}$. El discurso judicial de esta naturaleza ayuda a contrarrestar las tendencias despolitizantes de la adjudicación judicial al colocar las necesidades en discusión en un marco histórico y social más amplio de injusticia sistémica.

Además, los Tribunales pueden ayudar a cuestionar los estereotipos y las percepciones existentes sobre el papel de las prestaciones sociales en la sociedad. Ejemplo de esto es la forma en que la jueza Mokgoro, en el caso Khosa, trastoca el discurso habitual de que la asistencia social genera dependencia del Estado al subrayar el papel que desempeña en aliviar la carga de las comunidades pobres y fomentar la dignidad de los residentes permanentes ${ }^{97}$. Por último, el discurso del Tribunal puede servir como recordatorio permanente de que la reparación de la pobreza y la desigualdad son cuestiones de moralidad política y de responsabilidad social colectiva. Nuevamente, esto está ejemplificado en el caso Khosa en la siguiente observación de la jueza Mokgoro, cuando señala que:

"Compartir la responsabilidad de los problemas y las consecuencias de la pobreza de manera igualitaria, como una comunidad, representa el grado en el cual los miembros más adinerados de la comunidad consideran el bienestar mínimo de los pobres como algo relacionado con su propio bienestar y el bienestar de la comunidad en su conjunto. En otras palabras, las decisiones acerca de la asignación de beneficios públicos representan la medida en la cual se trata a la gente pobre como miembros de igual valor dentro de una sociedad" ${ }^{\prime 98}$.

En este sentido, el papel de los Tribunales consiste en mantener vivo en la conciencia pública el gran abismo que existe entre la visión de una sociedad justa reflejada en la Constitución y la realidad social. Como hizo notar Langa ACJ (como se Ilamaba entonces) en Modderklip:

"El hecho de que la pobreza y la falta de vivienda continúen acosando a muchos sudafricanos es un recordatorio penoso del abismo que debe ser salvado antes de poder lograr el ideal constitucional de establecer una sociedad basada en la justicia social y una mejor calidad de vida para todos los ciudadanos" ${ }^{\prime \prime 9}$. 
Mediante un discurso de esta naturaleza, los tribunales ayudan a contrarrestar los "retrocesos en el reconocimiento" ("recognition blacklash") que trae aparejada la entrega de beneficios sociales a los pobres.

\section{d) Transformación de las reglas subsidiarias del derecho consuetudinario}

En una economía de mercado, las reglas subsidiarias del derecho consuetudinario regulan el acceso y la distribución de los recursos. Danie Brand arguye que:

"Aunque el desarrollo de derechos socioeconómicos constitucionales con el fin de establecer nuevos y singulares remedios constitucionales es un esfuerzo importante en sí, para explorar el pleno potencial transformador de los derechos socioeconómicos es crucial también mantener un compromiso crítico sostenido con las reglas subsidiarias del derecho consuetudinario" 100 .

Como muestran los casos relativos a desalojos y carencia de vivienda, los derechos sociales han ayudado a deconstruir las ideas jerárquicas y absolutas de los derechos de propiedad ${ }^{101}$. Los intereses de la gente pobre en cuanto a la protección de sus hogares y a evitar la falta de vivienda se han convertido en un factor altamente pertinente en los casos de desalojo, con lo cual la propiedad del inmueble ha dejado de ser la carta de triunfo por excelencia ${ }^{102}$. En otros aspectos, los tribunales se han mostrado menos dispuestos a transformar las reglas del derecho consuetudinario a la luz de los compromisos con los derechos socioeconómicos ${ }^{103}$.

Aunque escapa al objetivo de este artículo, no se alcanzará el potencial transformador de la jurisprudencia sobre derechos sociales sin que existan formas más extendidas de procesos y prácticas de adjudicación que permitan hacerlos más accesibles y participativos. Tales reformas incluirían el logro del acceso equitativo a servicios legales de calidad, mecanismos perfeccionados para la implementación de los fallos sobre derechos sociales y la transformación de la cultura e ideología judicial ${ }^{104}$.

\section{Conclusión}

Concluyendo, se puede decir que es probable que exista una tensión persistente entre las tendencias despolitizantes de la adjudicación de derechos sociales y su potencial de transformación social. Quienes estén involucrados en litigios de derechos sociales deben tener presente esta tensión y tratar de minimizar las tendencias despolitizantes junto con aumentar al máximo las posibilidades del potencial transformador.

La obtención de beneficios sociales afirmativos mediante litigio puede crear un terreno fértil para una mayor movilización en torno a reformas más profundas. Una jurisprudencia sustantiva sobre derechos sociales puede facilitar "reformas no reformistas" y propiciar la transformación social en Sudáfrica. En particular, puede servir para incrementar la capacidad de participación de los que viven en situación de pobreza y 
exponer la naturaleza socialmente construida de la pobreza y la desigualdad. En el mejor de los casos, debiera servir de recordatorio constante de nuestro compromiso constitucional en cuanto a establecer una sociedad basada en la justicia social y facilitar la inclusión de la voz de los marginados en el debate de qué es lo que se requiere para lograr una sociedad de tales características.

Sin embargo, no podemos dar por sentado que se seguirá esta trayectoria transformadora. La exploración de la base teórica de los conceptos fundamentales de nuestra democracia constitucional, tales como la justicia social y la transformación, pueden orientarnos y ayudarnos a encontrar el camino.

\section{Notas}

1 Este artículo se basa en el discurso inaugural que la autora pronunció el 4 de octubre de 2005 en la Facultad de Derecho de la Universidad Stellenbosch. El artículo completo se publicará en Stellenbosch Law Review, 2006.

2 Véase Soobramoney v Minister of Health, KwaZulu-Natal 1997 (12) BCLR 1696 (CC), párrafo 8.

3 Karl Klare "Legal Culture and Transformative Constitutionalism", 1998, SAJHR 146. Klare describe el constitucionalismo transformador como un "proyecto a largo plazo de promulgación, interpretación y aplicación constitucional comprometida (evidentemente no en forma aislada sino en un contexto histórico de desarrollo político apropiado) con el fin de transformar las instituciones políticas y sociales y las relaciones de poder de un país, orientándolas en una dirección democrática, participativa e igualitaria", p. 150; C. Albertyn y B. Goldblatt, "Facing the Challenges of Transformation: Difficulties in the Development of an Indigenous Jurisprudence of Equality", 1998, SAIHR 248, 249; André van der Walt "Tentative urgency: sensitivity for the paradoxes of stability and change in the social transformation decisions of the Constitutional Court", 2000, 16 SA Public Law 1; H. Botha, "Metaphoric reasoning and transformative constitutionalism", 2003, TSAR 20; D. Moseneke, "Transformative adjudication", 2002, 18 SAJHR 309.

4 S vs. Makwanyane 1995 (6) BCLR 665 (CC), párrafo 262 (per Mahomed J); Bato Star Fishing (Pty) Ltd v Minister of Environmental Affairs and Tourism and Others 2004 (7) BCLR 687 (CC); Minister of Finance and Another vs. Van Heerden 2004 (11) BCLR 1125 (CC); Rates Action Group vs. City of Cape Town 2004 (12) BCLR 1328 (C), párrafo 100.

5 Sección 1.

6 “Transformative Adjudication", 2002, SAJHR 309, 314. Véase también: Investigating Directorate: Serious Economic Offences and Others vs. Hyundai Motor Distributors (Pty) Ltd and Others 2000 (10) BCLR 1079 (CC), párrafo 21; Government of the Republic of South Africa and Others vs. Grootboom and Others 2000 (11) BCLR 1169 (CC) [en adelante "Grootboom"], párrafo 1; Bel Porto School Governing Body and Others vs. Premier, Western Cape and another 2002 (9) BCLR 891, párrafo 6; Minister of Finance and Another vs. Van Heerden 2004 (11) BCLR 1125 (CC), párrafo 25; President of RSA and Another vs. Modderklip Boerdery (Pty) Ltd and Others 2005 (8) BCLR 786 (CC), párrafo 55.

7 Esta lucha no ha sido documentada integralmente. Una descripción abreviada aparece en S. Liebenberg y K. Pillay (eds) Socio-economic Rights in South Africa: A Resource Book, 2000, pp. 19-20.

8 En Government of the Republic of South Africa and Others vs. Grootboom and Others 2000 (11) BCLR 1169 (CC) (en adelante "Grootboom"), el Tribunal recalcó que "la esfera nacional del gobierno debe asumir la responsabilidad de garantizar que las leyes, las políticas, los programas y las estrategias sean adecuados como para cumplir con las obligaciones del Estado establecidas en la Sección 26", párrafo 40.

9 Pieterse, M., "Coming to Terms with Judicial Enforcement of Socio-Economic Rights" 397-398; Liebenberg, S., "Social and Economic Rights: A Critical Challenge" en Liebenberg, S. (ed.) The Constitution of South Africa from a Gender Perspective, 1995, pp. $79,84$. 
10 Como señalaron Scott y Maklem:

"Quizás la razón más poderosa para incluir ciertos derechos económicos y sociales radica en que, al elevar a rango constitucional la mitad de los derechos humanos en la ecuación, los sudafricanos estarían plasmando en la Constitución solamente parte de lo que constituye una persona plena. Una Constitución que sólo contiene derechos civiles y políticos proyecta una imagen de humanidad truncada. Simbólicamente, pero aun brutalmente, excluye aquellos segmentos de la sociedad para quienes la autonomía significa muy poco si no va acompañada de la satisfacción de necesidades básicas". C. Scott y P. Maklem, "Constitutional Ropes of Sand or Justiciable Guarantees? Social Rights in a New South African Constitution", 1992, Univ. of Penn LR 1, 29.

11 André van der Walt también señala que la fuerza de la interpretación de Frank Michelman al convertir una obligación moral, surgida de la extrema necesidad en un deber constitucional, "radica en que la teoría y la práctica sociales no quedan limitadas a un discurso sobre necesidades, sino que se realizan al interior del discurso tradicionalmente poderoso de los derechos". "A South African Reading of Frank Michelman's Theory of Social Justice", en Henk Botha, André van der Walt y Johan van der Walt (eds.) Rights and Democracy in a Transformative Constitution, 2004, p. 196.

12 N. Haysom, "Constitutionalism, Majoritarian Democracy and Socio-Economic Rights", 1992, SAJHR 451, 461.

13 Este es el principal organismo responsable de supervisar las obligaciones de los Estados-Partes bajo el Pacto Internacional sobre Derechos Económicos, Sociales y Culturales (1966). En su Observación General № 3, el Comité señaló que "es de la opinión de que corresponde a cada Estado-Parte una obligación mínima de asegurar la satisfacción de por lo menos niveles esenciales de cada uno de los derechos. Así, por ejemplo, un Estado-Parte en el que un número importante de individuos está privado de alimentos esenciales, de atención primaria de salud esencial, de abrigo y vivienda básicos o de las formas más básicas de enseñanza, prima facie no está cumpliendo sus obligaciones en virtud del Pacto... Para que cada Estado Parte pueda atribuir su falta de cumplimiento de las obligaciones mínimas a una falta de recursos disponibles, debe demostrar que ha realizado todo esfuerzo para utilizar todos los recursos que están a su disposición en un esfuerzo por satisfacer, con carácter prioritario, esas obligaciones mínimas". Observación General № 3 (Quinto período de sesiones, 1990) La índole de las obligaciones de los Estados-Partes (párrafo 1 del artículo 2 del Pacto) UN doc. E/1991/23, párrafo 10. Para ver una aplicación de este concepto en el contexto de los derechos específicos protegidos por el Pacto, véanse Observación General № 12 (Vigésimo período de sesiones 1999) El derecho a una alimentación adecuada (Artículo 11 del Pacto) UN doc. E/2000/22, párr. 17; Observación General № 14 (Vigésimo segundo período de sesiones, 2000) El derecho al disfrute del más alto nivel posible de salud (Artículo 12 del Pacto) UN doc. E/C.12/2000/4, párrs. 43-47; Observación General № 15 (Vigésimo noveno período de sesiones) El derecho al agua (Artículos 11 y 12 del Pacto) UN doc. E/ C.12/2002/11, párrs. 37-38.

14 TAC, párr. 39.

15 Grootboom, párr. 38.

16 Grootboom, párrs. 32 y 33. En consecuencia, en el contexto del derecho a tener acceso a una vivienda adecuada, el Tribunal destacó el hecho de que las necesidades de los grupos cuya situación es diferente tienen necesidades distintas: "Hay quienes necesitan tierras; otros necesitan tanto tierras como viviendas; en tanto otros, necesitan asistencia económica". (Párr. 33).

17 TAC, párr. 35.

18 Sostuvo que "los Tribunales no cuentan con las facultades institucionales para llevar a cabo las exhaustivas investigaciones políticas y sobre los hechos que se requieren para determinar lo que debieran ser las "obligaciones básicas mínimas". (TAC párr. 37).

19 Grootboom, párr. 33; TAC, párr. 34

20 Véase Grootboom, párr. 41. Este es un modelo justificatorio del fin para los medios en el cual el Tribunal se plantea la pregunta básica de si se justifica una política o programa en particular. Se justificará "si está razonablemente relacionada con el objetivo constitucional de otorgar acceso a los derechos socioeconómicos pertinentes". Véase Danie Brand, "The Proceduralisation of South African Socio-economic Rights Jurisprudence, or "What are Socio-economic Rights For?" en Henk Botha, André van der Walt y Johan van der Walt (eds.) Rights and Democracy in a Transformative Constitution, 2004, 33 pp. 39-43. 
21

22 Grootboom párr. 43

23 Grootboom, párr. 36. Véase también TAC: "Hay una diferencia en las posiciones de quienes pueden pagar los servicios y aquéllos que no los pueden pagar. La política estatal debe tomar en cuenta tales diferencias". Párr. 70.

24 Grootboom, párr. 44 (énfasis agregado).

25 Grootboom: "En otras palabras, no hay una disposición explícita para facilitar el acceso a la ayuda temporal para gente que carece de acceso a tierras, que no tiene un techo, gente que está viviendo en condiciones insoportables y gente que está en una crisis debido a desastres naturales, tales como inundaciones, incendios o porque sus casas van a ser demolidas". Párr. 52.

26 TAC, párr. 73

27 TAC: "Hemos señalado que esta política no cumple con los estándares constitucionales, porque excluye a personas que razonablemente podrían ser incluidas en casos en que dicho tratamiento se justifica en términos médicos para evitar la transmisión del VIH de madre a hijo". Párr. 125.

28 TAC, párr. 125.

29 Grootboom, párr. 99.

30 TAC, párr. 135

31 Grootboom: "Ni la Sección 26 ni la Sección 28 otorgan el derecho a los demandantes a reclamar alojamiento o vivienda inmediatamente por la sola demanda", párr. 95; TAC: “...los derechos socioeconómicos de la Constitución no deberán interpretarse como el otorgamiento de un derecho a todos de exigir que se le entregue el mínimo básico". Párr. 34; Véase también: TAC párr. 39; Jaftha párr. 32-33 (distinción entre obligaciones negativas y positivas).

322004 (6) BCLR 569 (CC)

33 La legislación pertinente fue la Ley de Asistencia Social (Social Assistance Act 59) de 1992, y la Ley de Reforma de las Leyes de Bienestar (Welfare Laws Amendment Act 106) de 1997.

34 Khosa, párr. 78.

35 En vista de que el Estado ya había reconocido la inconstitucionalidad del requisito de ciudadanía en cuanto a las subvenciones a favor de la infancia, la inclusión de los residentes permanentes en las subvenciones sociales restantes implicaría un incremento inferior al $2 \%$ del costo actual de las subvenciones actuales. En consecuencia, el Tribunal concluyó que "el costo de incluir a los residentes permanentes en el sistema sólo representará una pequeña parte en proporción al costo total". (Párrafo 62).

36 Khosa, párr. 65 (énfasis agregado).

37 Khosa, párrs. 64-65.

38 Khosa, párr. 82.

39 Khosa: "Lo que distingue a este caso de los otros analizados previamente por este Tribunal es que, además de los derechos a la vida y la dignidad, el sistema de seguridad social implementado por el Estado destinado a cumplir con sus obligaciones en virtud de la Sección 27 de la Constitución plantea la pregunta de la prohibición de la discriminación injusta". Párrafo 44. Véase también párr. 49.

40 Khosa, párr. 83.

41 Khosa, párr. 84.

42 Khosa, párr. 86-89; The Order (párr. 98).

43 Jaftha, párr. 34.

44 Grootboom, párr. 34; TAC, párr. 46.

45 La Sección 26(3) señala: "Nadie podrá ser desalojado de su vivienda ni se podrá demoler su vivienda sin mediar una orden del Tribunal, la cual será elaboraba luego de considerar todas las circunstancias pertinentes. Ninguna legislación podrá permitir desalojos arbitrarios".

46 PIE revocó la antigua Ley de Prevención de Ocupación Ilegal de Tierras (Prevention of Illegal Squatting Act 52) de 1951 mencionada anteriormente. Otra pieza clave de legislación que pone en vigencia a la Sección 26(3) es la Ley de Extensión de la Seguridad de Tenencia (Extension of Security of Tenure Act 62- ESTA) de 1997. 
47 Port Elizabeth Municipality vs. Various Occupiers 2004 (12) BCLR 1268 (CC).

48 PE Municipality, párr. 21.

49 PE Municipality, párr. 28.

50 PE Municipality: La existencia de un programa de vivienda "diseñado para albergar al mayor número de personas sin casa en el menor plazo de tiempo de la forma más eficiente en función de los costos" no es suficiente para determinar "si se debiera realizar efectivamente una orden de desalojo y bajo qué condiciones en un caso determinado". Párrafo 29.

51 PE Municipality, párrs. 39-47, párr. 43.

52 PE Municipality, párr. 23.

53 PE Municipality, párr. 26.

54 PE Municipality, párr. 58.

55 President of RSA and Another vs. Modderklip Boerdery (Pty) Ltd and Others 2005 (8) BCLR 786 (CC).

56 Modderklip, párr. 9.

57 Modderklip, párr. 26. Esto contrasta con el fallo SCA en el caso: President of the Republic of South Africa and Others vs. Modderklip Boerdery (Pty) Ltd 2004 (8) BCLR 821. Además, SCA había sostenido que también se habían violado las disposiciones de igualdad en virtud de la Sección 9(1) y (2) de la Constitución.

58 Modderklip, párrs. 43-51.

59 Modderklip, párr. 49

60 Modderklip, párr. 49.

61 Modderklip, párr. 49.

62 Modderklip, párr. 68, Order, párr. 3(c).

63 2005(1)BCLR 78 (CC).

64 Jaftha, párr. 12.

65 Jaftha, párr. 32.

66 Jaftha, párrs. 31-34.

67 Además, la Sección 36 requiere una evaluación del grado al cual los objetivos de la limitación son compatibles con "una sociedad democrática y abierta que se basa en la dignidad humana, la igualdad y la libertad". También incorpora una evaluación rigurosa de la proporcionalidad, incluyendo la disponibilidad de "medios menos restrictivos" para lograr los fines del Estado (Sección 36(1)(e)).

68 Jaftha, párr. 34.

69 Jaftha, párrs. 35-51.

70 Jaftha, párrs. 52-67.

71 Jaftha, párr. 56.

72 Jaftha, párr. 59.

73 D. Davis, "Socio-economic rights in South Africa: The record of the Constitutional Court after 10 years" (2004) 5 ESR Review 3, 5. Como señalan Currie y De Waal: "Una característica de una norma legal es que se otorga considerable discreción de interpretación al adjudicador responsable de su aplicación, motivo por lo cual no especifica el resultado por anticipado". The Bill of Rights Handbook (5 $5^{\mathrm{a}}$ ed., 2005) 579.

74 Véase la crítica de Sandy Fredman del enfoque adoptado por la Corte Suprema de Canadá en el caso Gosselin vs. Quebec (Ministro de Justicia) 2002 SCC 84 en la revisión de los reglamentos de seguridad social en Quebec que discriminaban en la entrega de beneficios de asistencia social a personas menores de 30 años. S. Fredman, "Providing Equality: Substantive Equality and the Positive Duty to Provide" (2005) 21 SAJHR 163. Pieterse también manifiesta dudas acerca de si es adecuada "la naturaleza relativamente abstracta y abierta de la investigación sobre la razonabilidad para desarrollar una jurisprudencia sobre derechos socioeconómicos en resonancia con el derecho internacional y los objetivos transformadores del orden constitucional". "The Judicial Enforcement of Socio-Economic Rights" 410. Bilchitz critica la revisión de la razonabilidad por no lograr desarrollar adecuadamente el contenido de las obligaciones que imponen los diferentes derechos y observa que actualmente "pareciera actuar como sustituto de cualquier cosa que el Tribunal considera como características desea- 
bles de la política estatal". "Towards a Reasonable Approach to the Minimum Core" 19 SAJHR $9-10$.

75 S. Liebenberg, "Enforcing Positive Socio-Economic Rights Claims: The South African Model of Reasonableness Review", en J. Squires, M. Langford y B. Thiele (eds), The Road to a Remedy: Current Issues in the Litigation of Economic, Social and Cultural Rights, The University of New South Wales Press, Sydney, 2005, p. 73.

76 Grootboom: "La razonabilidad se debe determinar conforme a los hechos en cada caso", párrs. 43-44. Párr. 92.

77 Véase la descripción de Mark Heywood de las estrategias de TAC en el caso MTCT: "Preventing Mother-to-Child HIV Transmission in South Africa: Background, Strategies and Outcomes of the Treatment Action Campaign Case Against the Minister of Health", 2003, SAJHR, 278.

78 Véase, por ejemplo, la presentación (en línea) de la sociedad civil sobre el plan operativo para el inicio del programa antirretroviral denominado "A People-Centred ARV Programme" en el sitio web: www.tac.org.za/Documents/TreatmentPlan/ FullFinalSubmissiontoARVTaskTeam.doc

79 En este sentido, la jurisprudencia de los derechos socioeconómicos converge con la jurisprudencia de igualdad sustantiva, particularmente en lo que se refiere a la prueba de discriminación injusta y el enfoque del Tribunal a la igualdad compensatoria: Harksen vs. Lane $N^{\circ} 1998$ (1) SA 300 (CC), párr. 53; Minister of Finance and Another vs. Van Heerden 2004 (11) BCLR 1125 (CC), párr. 25-32. Para una discusión de esta convergencia, véase: P. de Vos, "Grootboom, The Right of Access to Housing and Substantive Equality as Contextual Fairness" (2001) SAJHR 258.

80 Fraser, Nancy, "Social Justice in the Age of Identity Politics: Redistribution, Recognition, and Participation" en Fraser, N. y Honneth, A. Redistribution or Recognition? A PoliticalPhilisophical Ex change, Londres, New York, Verso, 2003.

81 PE Municipality párr. 38.

82 Grootboom: "Significa que la posibilidad de acceso debe facilitarse en forma progresiva: es necesario examinar las cargas administrativas, operativas y financieras y, cuando sea posible, disminuirlas en el transcurso del tiempo". Párr. 45.

83 Grootboom, párr. 45 citando en forma aprobatoria la Observación General № 3 del Comité de la ONU sobre Derechos Económicos, Sociales y Culturales, párr. 9 (énfasis agregado).

84 Estas incluyen la Ley de Compensación por Accidentes y Enfermedades Laborales (Occupational Injuries and Diseases Act -COIDA- Act Nº 130) de 1992, la Ley de Seguro de Desempleo (Unemployment Insurance Act 63) de 2001 y una serie de sistemas de pensión otorgados por los empleadores.

85 Estas subvenciones sociales se pagan en virtud de la Ley de Asistencia Social (Social Assistance Act $N^{\circ}$ 59) de 1992 recientemente reemplazada por la Social Assistance Act № 13 de 2004.

86 La definición de "niño" en la Sección 28(3) de la Constitución señala que se trata de "una persona menor de 18 años de edad".

87 S. Liebenberg, "The Right to Social Assistance: The Implications of Grootboom for Policy Reform in South Africa" (2001) 17 SAJHR 232.

88 J. Streak, "Government's social development response to children made vulnerable by HIV/AIDS: Identifying gaps in policy and budgeting" IDASA Occasional Paper, 9 september 2005, 21.

89 Sección 27(1)(c) le otorga a todas las personas el derecho a tener acceso a "la seguridad social incluso, si no son capaces de proporcionarse el sustento y el de sus cargas familiares, asistencia social adecuada".

90 La única excepción es la Pensión de Asistencia Social por Penuria (Social Relief of Distress Grant-SROD) que se otorga en virtud de la Ley de Asistencia Social (Social Assistance Act). Sin embargo, esta subvención tiene un límite máximo de 3 meses sucesivos, tiene requisitos de elegibilidad restrictivos y no está bien administrada. Véanse los Reglamentos de la Ley de Asistencia Social 13 de 2004, № R. 162, Notificación del Gobierno 27316, 22 de febrero de 2005, ítemes 9, 14, 15 y 23.

91 Observación General № 14, párr. 43(f); Observación General № 15, párr. 37(f).

92 Véase la discusión en la nota 185 y el texto que la acompaña en la versión completa de este artículo. 
* Nota del traductor: la versión en inglés dice supervisory remedies, para lo cual la autora ha explicado que se trataría del caso en que una corte ordena al Estado informar acerca de las medidas que está tomando para dar cumplimiento a una orden emitida por ella y luego le ordena tomar medidas adicionales hasta que la corte considera que el derecho constitucional en cuestión se ha hecho realmente efectivo.

93 Sobre medidas de supervisión en el contexto de litigios por derechos socioeconómicos, véase W. Trengove, "Judicial Remedies for Violations of Socio-economic Rights (1999) ESR Review 8. En cuanto a críticas de la reticencia del Tribunal para otorgar medidas de supervisión, véase D. Bilchitz "Towards a Reasonable Approach to the Minimum Core: Laying the Foundations for Future Socio-economic Rights Jurisprudence, 2003, SAJHR 1 , 23-4; K. Pillay, "Implementing Grootboom" 275 -6; Heywood, "Preventing Mother-toChild HIV Transmission in South Africa" 311 -12.

94 Grootboom, párr. 44.

95 Al comentar sobre la teoría basada en las necesidades, desarrollada por Frank Michelman, André van der Walt señala una implicación retórica y teórica importante de este enfoque:

“... [C]oloca de cabeza la tradicional estrategia teórica basada en derechos (rights-based theory) al centrarse en la necesidad individual, la marginalidad, la debilidad y la impotencia en lugar de basarse en el poder legal. La garantía constitucional no se basa en lo que uno posee, sino que en las carencias y necesidades del individuo, en aquello que lo vuelve débil".

De este modo, su teoría rechaza una noción puramente estabilizadora, conservadora de la justicia social, reemplazándola con normas y principios correspondientes a la evaluación de resultados distribucionales: A. van der Walt "A South African Reading of Frank Michelman's Theory of Social Justice" en H. Botha, A. van der Walt y J. van der Walt (eds.) Rights and Democracy in a Transformative Constitution, 2004, 163, 193. Véase también 198-199. Respecto de la teoría basada en las necesidades propuesta por Frank Michelman, véase F. I. Michelman, "The Supreme Court 1968 Term-Foreward: On Protecting the Poor through the Fourteenth Amendment", 1969, Harv LR 7; "Welfare Rights in a Constitutional Democracy", 1979, Wash LQ 659.

96 PE Municipality, párrs. 8-23.

97 Khosa, párr. 76.

98 Khosa, párr. 74 (notas al pie omitidas).

99 Modderklip, párr. 36 (notas al pie omitidas).

100 Brand, "Introduction to socio-economic rights in the South African Constitution" 39. El impacto de los derechos sociales sobre el derecho consuetudinario también puede tener lugar a través de la promulgación de legislación que ponga en vigencia dichos derechos, por ejemplo, PIE. Véase Ndlovu vs. Ngcobo; Bekker vs. Jika 2003 (1) SA 113 (SCA): "Algunos estimarán lamentable que el Poder Legislativo, en forma casi imperceptible e indirecta, haya eliminado derechos consagrados por el derecho consuetudinario para promover derechos sociales. Otros señalarán que los derechos sociales efectivamente tienden a afectar o a impactar los derechos consuetudinarios, a menudo en forma dramática". (Párrafo 16). Véase también: T. Roux "Continuing and change in a transforming legal order:The impact of section 26(3) of the Constitution on South African law", 2004, 121 SALJ 466.

101 Ndlovu vs. Ngcobo; Bekker vs. Jika 2003 (1) SA 113 (SCA). Ver también nota № 100.

102 A. van der Walt, "Exclusivity of Ownership, Security of Tenure, and Eviction Orders: A Critical Evaluation of Recent Case Law", 2002, 18 SAJHR 371.

103 Véase, por ejemplo, Brisley vs. Drotsky 20024 SA 1 (SCA); Afrox Healthcare (Pty) Ltd vs. Strydom 20026 SA 21 (SCA); D. Brand "Introduction to socio-economic rights in the South African Constitution", en D. Brand y C. Heyns (eds.) Socio-economic Rights in South Africa (2005) 1, 41-42.

104 Para ver una discusión de los problemas de ideología y cultura judiciales en el contexto de la adjudicación de derechos socioeconómicos, véase M. Pieterse "Coming to Terms with Judicial Enforcement of Socio-economic Rights" 396-399. 